(C) 2000 International Press

Adv. Theor. Math. Phys. 3 (2000) 479-494

\title{
A Comment on
}

\section{Nonsupersymmetric Fixed}

\section{Points and Duality at large $\mathrm{N}$}

\author{
Micha Berkooz and Anton Kapustin
}

\author{
School of Natural Sciences \\ Institute for Advanced Study \\ Princeton, NJ 08540, USA \\ berkooz@sns.ias.edu \\ kapustin@sns.ias.edu
}

\begin{abstract}
We review some of the problems associated with deriving field theoretic results from nonsupersymmetric AdS, focusing on how to control the behavior of the field theory along the flat directions. We discuss an example in which the origin of the moduli space remains a stable vacuum at finite $N$, and argue that it corresponds to an interacting CFT in three dimensions. Associated to this fixed point is a statement of nonsupersymmetric duality. Because $1 / N$ corrections may change the global picture of the RG flow, the statement of duality is much weaker than in the supersymmetric case.
\end{abstract}

e-print archive: http://xxx.lanl.gov/hep-th/9903195 


\section{Introduction}

The AdS/CFT correspondence [13], [19] is a powerful tool for studying the large $N$ limit of field theories. By now a significant number of matches has been made between the dynamics of gauge theories and the dynamics of supergravity in the corresponding backgrounds. For the most part this analysis has been carried out in a supersymmetric setting.

An interesting question is whether one can use gravity to understand the dynamics of nonsupersymmetric conformal field theories at large $\mathrm{N}$. To answer this question one is led to study string theory/M-theory backgrounds of the form $A d S_{p} \times M_{q}$ where $M_{q}$ is a compact manifold which breaks supersymmetry (either via orbifolding a supersymmetric manifold [11], or by other means [5]). Another approach (related to the previous one [15]) uses type 0 string theory [12].

When discussing nonsupersymmetric theories one usually appeals to classical 11D supergravity (i.e., the leading term in the momentum expansion) or to classical string theory, both of which correspond to $N=\infty$. In trying to extend the discussion to large but finite $\mathrm{N}$ one generically runs into problems. In [5] the following two problems were discussed:

- If for $N=\infty$ there are fields whose masses are at the Breitenlohner-Freedman unitarity bound, then these masses might be pushed below the bound by $1 / N$ corrections.

- If there are massless fields (i.e. fields that correspond to marginal operators at $N=\infty$ ) which are invariant under all the symmetries, then $1 / N$ corrections may shift their VEVs significantly and there may not be a stable vacuum for finite $N$, or if such vacuum exists, it may be qualitatively different from the $N=\infty$ starting point.

It was shown in [5], however, that it is easy to construct models in which these problems do not arise.

Another problem which we will discuss in this paper is that of the fate of flat directions present at $N=\infty$. Many nonsupersymmetric 
gauge theories converge, in some formal sense at least, to a theory with sixteen supercharges as $N \rightarrow \infty$ [6], so in this limit the scalar potential has flat directions. These flat directions are typically lifted by $1 / N$ effects, as a result of which the fields are either driven away from the origin or attracted to the origin (or a combination of both in different directions). In the former case the vacuum at the origin is destabilized (in fact, the theory may not have any stable vacuum at all), while in the latter case the origin is at least perturbatively stable. In the latter case there is generically a mass gap, explaining why it is so hard to construct nonsupersymmetric CFTs when scalars are present (there are however examples of nonsupersymmetric fixed points with fermions in the weak coupling regime [2]). In this paper we will discuss a $2+1$ dimensional example in which the flat directions are lifted in a way which drives the fields to the origin, nevertheless the theory does not become massive and trivial there.

Before proceeding it is worth mentioning some open problems. The main open problem is that it is not clear whether the expansion around $N=\infty$ is only formal, or whether it can be used to really approximate the physics at finite $N$. In backgrounds that correspond to weakly coupled string theory there is a genus expansion which is an expansion in $1 / N$. If the contribution of each genus is finite then there is a valid $1 / N$ expansion. However, models in the perturbative stringy regime, for example those based on D3-branes, run into problem 2 (the dilaton is always a dangerous massless field). In the strong coupling regime (M-theory or type IIB string theory near its self-dual points) it is not clear whether quantum corrections are small. More on this point will appear in [4].

Another open problem is the issue of nonperturbative instabilities which describe tunneling in the bulk. Presumably these effects are exponentially small at large $N$. Not much is known about such instabilities (see however [14]), and we will not change this situation here. 


\section{The Example}

The example that we will focus on is that of M-theory on ${ }^{1} A d S_{4} \times \mathbf{S}^{7} / \mathbb{Z}_{2}$. This background is obtained by probing different kinds of $\mathbb{R}^{8} / \mathbb{Z}_{2}$ orbifolds of M-theory with either M2-branes or anti-M2-branes.

The two kinds of $\mathbb{R}^{8} / \mathbb{Z}_{2}$ orbifolds differ by the charge of the singularity. The first one, which we call the A-orbifold, has membrane charge $-1 / 16$, while the other one, which we will call the B-orbifold, has charge 3/16 [17]. Both orbifolds preserve sixteen supercharges, the same supercharges as those preserved by an M2-brane parallel to the orbifold plane. Hence probing the orbifold singularities by M2-branes yields $\mathcal{N}=8$ field theories in three dimensions. Supersymmetry implies that when the charge of the orbifold singularity is positive (relative to that of the M2-brane) the long range gravitational field of the singularity is as if it had a positive mass; contrary-wise, if the charge is negative, then the mass is negative (this, for example, can be deduced from the cancelation of forces between the M2-brane and the singularity).

For both singularities the near-horizon geometry in the limit of large number of probes $N$ is $A d S_{4} \times \mathbb{R P}^{7}$. The only difference between the two backgrounds is the torsion class $[18]$ in $H^{4}\left(\mathbb{R P}^{7}, \mathbb{Z}\right)=\mathbb{Z}_{2}$ which specifies how a membrane propagating in this background is to be quantized [18], [17], [3]. The A-singularity corresponds to a trivial torsion class, while the B-singularity corresponds to a nontrivial one. In the large $N$ limit the curvature is small, and M-theory on $A d S_{4} \times \mathbb{R P}^{7}$ reduces to supergravity on the same background. Since supergravity is insensitive to the torsion, the supergravity spectrum will be exactly the same for the two backgrounds. In this limit, the difference in the torsion class becomes visible only if one considers solitonic objects (M2-branes and M5-branes) wrapping nontrivial cycles of $A d S_{4} \times \mathbb{R P}^{7}$.

Similarly we can probe the A and B singularities with anti-M2branes. This yields models without any supersymmetry. The near horizon geometry in this case is the "skew-whiffed" $A d S_{4} \times \mathbb{R P}^{7}$ [8]. The usual logic of the AdS/CFT correspondence leads to the conclusion that M-theory on a "skew-whiffed" $A d S_{4} \times \mathbb{R P}^{7}$ describes a nonsupersymmetric CFT on the boundary. The backgrounds obtained from the

\footnotetext{
${ }^{1}$ The spectrum is related to that of $A d S_{4} \times S^{7}$. The spectrum of the latter is computed in [7] and compared to field theory expectations in [1].
} 
A and B singularities differ only by a torsion class which does not affect the Kaluza-Klein spectrum.

Both $\mathrm{A}$ and $\mathrm{B}$ singularities can be regarded as a strong-coupling limit of certain orientifold backgrounds in IIA string theory [17], [3]. An $O 2^{-}$plane lifts to an M-theory background of the form $\left(\mathbb{R}^{7} \times \mathbf{S}^{1}\right) / \mathbb{Z}_{2}$ which has two orbifold singularities of type A. An $\mathrm{O}^{+}$plane lifts to the same orbifold, except that one singularity is of type A, and the other one is of type B. Finally, an $\widetilde{O 2}^{+}$plane (which is an $\mathrm{O}^{-}$plane with a half-D2-brane stuck to it) lifts to a pair of B-singularities. These IIA backgrounds can be probed with (anti-)D2 branes, which lift to (anti)M2-branes of M-theory. Thus the $\mathcal{N}=8$ CFTs described by M-theory on $A d S_{4} \times \mathbb{R P}^{7}$ are related to $\mathcal{N}=8$ gauge theories on D2-branes, while the $\mathcal{N}=0$ CFTs described by M-theory on the "skew-whiffed" $A d S_{4} \times \mathbb{R P}^{7}$ are related to the gauge theories on anti-D2-branes. The precise nature of this relation will be discussed in sec-4. In this paper we will focus on the $\mathcal{N}=0$ case.

Reference [5] discusses some aspects of supergravity on the "skewwhiffed" $A d S_{4} \times \mathbb{R P}^{7}$. It was shown there that the Kaluza-Klein spectrum has neither massless charged scalars, nor modes saturating the Breitenlohner-Freedman bound. As explained in the introduction, this implies that the "skew-whiffed" $A d S_{4} \times \mathbb{R P}^{7}$ avoids some immediate problems of nosupersymmetric compactifications. In the next section we will address another potential problem associated with the presence of flat directions at infinite $N$. We will argue that for the B-singularity the potential generated along the flat directions at large but finite $N$ does not change the vacuum significantly. The model corresponding to the A-singularity is apparently destabilized by $1 / N$ corrections.

\section{Lifting of the flat direction}

We are therefore interested in discussing anti-M2-branes probing an $A$ or $B \mathbb{R}^{8} / \mathbb{Z}^{2}$ singularity. Equivalently one may consider M2-branes probing the charge-conjugated singularities which we will call $\bar{A}$ and $\bar{B}$. In this section we will use the latter viewpoint.

At leading order in $N$ there are flat directions which correspond to 
moving the branes away from the singularity and away from each other ${ }^{2}$. This can be seen in several ways, but in general one expects [6] that at $N=\infty$ the structure of the flat directions is the same as in the corresponding $\mathcal{N}=8$ theory.

To obtain some information about the potential along the flat directions one can do a long distance M-theory computation: one can place the branes at a distance $r \gg l_{p}$ from the singularity and determine, based on the charge and mass of the singularity, whether there is an attractive or repulsive force between the branes and the singularity. This computation has little to do with field theory, since the branes are in the asymptotically flat region. However, because this computation depends on the mass and charge of the singularity in the same way as the correct near horizon computation, it distinguishes correctly between attractive and repulsive potential.

Using this approach one can also see that the potential is subleading in $1 / N$. The leading term in the long distance computation $\left(r \gg l_{p}\right)$ is nominally of order $N^{2}$ (coming from all pairwise interactions between the branes), but because this is the same as in the $\mathcal{N}=8$ theory it is $N^{2} \times 0=0$. On the other hand, the interaction between the singularity and the branes is of order $N$, because there is only one singularity.

The computation that we would like to do is to check the stability of the AdS to fragmentation along the flat directions in the near horizon geometry. The idea is to separate the branes into several clusters and compute the potential as a function of separation. For simplicity we will focus on the case of a single cluster away from the singularity (i.e., two clusters which are the images of each other).

\subsection{The approximate solution along the flat direc- tions}

We will start with the supergravity solution representing two clusters of M2-branes in flat space and then orbifold this solution. The metric for several parallel D3-branes in flat space was written in [13] and it is

\footnotetext{
${ }^{2} \mathrm{We}$ are referring to the flat directions of the fixed point theory in the IR rather than to those of the UV theory which flows to it.
} 
straightforward to generalize the ansatz [9] to M2-branes:

$$
\begin{gathered}
d s^{2}=f^{-2 / 3} d x^{2}+f^{1 / 3}\left(d r^{2}+r^{2} d \Omega^{2}\right) \\
G_{x^{0} x^{1} x^{2} r^{i}} \propto \partial_{r^{i}} f^{-1}(r),
\end{gathered}
$$

where $G$ is the 4-form field strength and $f$ is an harmonic function of the 8 -vector $r$. To obtain the situation with two clusters each containing $N$ M2-branes we set

$$
f(r)=\frac{N l_{p}^{6}}{|r-a|^{6}}+\frac{N l_{p}^{6}}{|r+a|^{6}},
$$

where the 8-vector $a$ is the position of the cluster. From the field theory point of view it is convenient to do a rescaling $u^{i}=r^{i} / l_{p}^{\frac{3}{2}}[13]$.

Next we want to orbifold this background. Orbifolding introduces an $\mathbb{R}^{8} / \mathbb{Z}_{2}$ singularity at $r=0$. To facilitate the analysis of this background it is convenient to further rescale the coordinates so that the metric near the origin is the canonical flat metric on $\mathbb{R}^{11}$ :

$$
y^{i}=\left(\frac{2 N l_{p}^{6}}{a^{6}}\right)^{-\frac{1}{3}} x^{i}, z^{i}=\left(\frac{2 N l_{p}^{6}}{a^{6}}\right)^{\frac{1}{6}} r^{i},
$$

after which the metric and the 4-form are given by the same ansatz but with the following harmonic function: $\hat{f}$ :

$$
\hat{f}=\frac{1 / 2}{\left|n-\frac{z}{(2 N)^{1 / 6} l_{p}}\right|^{6}}+\frac{1 / 2}{\left|n+\frac{z}{(2 N)^{1 / 6} l_{p}}\right|^{6}},
$$

where $n$ is a unit 8-vector in the direction of $a$.

Since the metric near the origin is the canonical one, and for large $N$ all curvatures and field strengths are small there, it is easy to insert the fields of the $\mathbb{Z}_{2}$ singularity at $z=0$. One can identify the following regions in the orbifolded background:

1. $z^{2}<l_{p}^{2}$ : inside this region the curvature and the field strength produced by the singularity are large. Our knowledge of this this region is not better or worse than that of the $\mathbb{R}^{8} / \mathbb{Z}_{2}$ singularity in flat space. The fields due to the clusters of M2-branes (the curvature and the 4form) are of order $1 / N^{\frac{1}{6}}$ there. 
2. The fields produced by the singularity and the fields produced by the branes are comparable when

$$
\frac{1}{z^{7}} \sim \frac{1}{N^{\frac{1}{6}}}
$$

At this point both are weak and can be treated using perturbation theory around flat space (locally).

3. At $z \sim N^{\frac{1}{6}} n$ we approach the cluster of M2-branes around which the space looks like $A d S_{4} \times \mathbf{S}^{7}$. This describes an $\mathcal{N}=8 \mathrm{IR}$ fixed point to which our theory flows along this flat direction.

In the region $z>l_{p}$, the fields produced by the singularity are small, and so are the fields of the original background. The gravity background is therefore under control, and furthermore, the corrections to the background due to the introduction of the singularity are small as well. In the following subsection we will extract the influence of this small correction on the potential along the flat directions.

\subsection{The potential along the flat directions}

We would like to know whether, upon the introduction of the singularity, there is a potential which drives the center of the cluster to the origin or repels it. This potential is subleading in the $1 / N$ expansion and can be easily computed if one neglects the back-reaction of the singularity on the rest of the geometry. We saw above that this approximation is valid for $z>l_{p}$.

Within this approximation the computation is straightforward. If we were allowed to choose the mass $(m)$ and charge $(Q)$ of the singularity arbitrarily (the charge is measured relative to the charge of the M2-branes), then there would be a line in the $Q-m$ plane, $Q=m$ in appropriate units, on which supersymmetry is preserved. A and $\mathrm{B}$ singularities correspond to two points on this line (A has negative charge, while $\mathrm{B}$ has positive charge). The points corresponding to the $\bar{A}$ and $\bar{B}$ singularities which break supersymmetry also have charges of opposite sign and lie on on the line $Q=-m$. Clearly the sign of the potential will change when going from one side of the line $Q=m$ to the other. Hence one of the SUSY-breaking singularities will attract the two clusters of branes, and the other will repel them. 
In more detail, the computation goes as the follows. When we take into account the singularity the action is

$$
\mathcal{L}=\mathcal{L}_{0}+m \int_{r=0} d^{3} x \sqrt{g_{\text {ind }}}+Q \int_{r=0} C^{(3)}
$$

where $\mathcal{L}_{0}$ is the usual action of $11 \mathrm{D}$ supergravity and $g_{\text {ind }}$ is the determinant of the induced metric on the plane $r=0$. The fields in $\mathcal{L}_{0}$ are the same as in the supersymmetric case, except for a two-fold identification due to orbifolding. The terms localized at $r=0$ are due to the mass and charge of the singularity. To compute the leading contribution to the potential in the no-back-reaction approximation one has to insert the ansatz (1) for the two symmetrically separated clusters into this action.

The terms that we are interested in are the kinetic terms for $a^{i}\left(x^{\mu}\right)$ (we allow $a$ to depend slowly on $x^{\mu}$ ) and the terms that encode the interaction of clusters with the singularity. The latter are proportional to $\int_{r=0} d x C^{(3)}$ (the gravitational term gives an equal contribution as can be seen by comparison with the supersymmetric case). This gives a term in the effective Lagrangian for $a$ of the form

$$
\frac{1}{N} \int d^{3} x\left(U^{i}\right)^{6}
$$

where $U$ is the field theory quantity with dimension $1 / 2\left(U^{i}=a^{i} / l_{p}^{3 / 2}\right)$.

The kinetic term is also easy to evaluate. The functional dependence is determined by spontaneously broken scale invariance to be proportional to

$$
\int d^{3} x\left(\partial_{\mu} U^{i}\right)^{2}
$$

The coefficient in front of this term is of order $N$. This can be seen by rescaling the coordinates $x$ so that the entire metric in the new coordinates is proportional to $N^{\frac{1}{3}}$. In this setup it is easy to obtain the $N$-scaling of $\mathcal{L}_{0}$ and therefore the $N$-scaling of the kinetic term.

The result of this computation is that for a singularity with negative charge $(\bar{B})$ there is an attractive potential along the flat directions, while for $\bar{A}$ the potential is repulsive. Furthermore, since the potential is suppressed by powers of $N$, it is small at large $N$, and the no-backreaction approximation is self-consistent. 


\section{Nonsupersymmetric Duality}

\subsection{Weakness of nonsupersymmetric duality}

The statement that we are after is that of IR duality, i.e., we would like to exhibit two distinct (weakly coupled) theories in the UV which flow in the IR to the fixed point described above. However, the duality that we obtain here will be considerably weaker than the one obtained in cases with higher supersymmetry.

\subsubsection{Field theory considerations}

The reason that the duality is weaker is the following. Let us first consider the case $N=\infty$. In this case the theory is a projection of the $\mathcal{N}=8$ theory, in the sense that its dynamics is the same as in the latter, except that we restrict our attention to a subset of operators [6]. The dynamics of the $\mathcal{N}=8$ theory is well understood [16] and it is known that at the origin of its moduli space it flows from a free UV fixed point to an interacting superconformal IR fixed point.

Consider now the $1 / N$ corrections to the RG flow. They are present everywhere along the RG trajectory. Such corrections, even though they are small at each point in the field theory parameter space, can change the global picture of the RG flow. Therefore they may change the statement that the theory flows from the gaussian fixed point in the UV to the interacting IR.

Nevertheless, even with $1 / N$ corrections taken into account, there exists an RG trajectory which ends at the IR fixed point and passes at a distance of order $1 / N$ from the gaussian fixed point. Therefore, if one wishes to "land" at the IR fixed point, one needs to fix a cutoff and add, besides the relevant perturbation that already exists in the $N=\infty$ theory, other operators with fine-tuned coefficients suppressed by powers of $1 / N$. In principle, at each order in $1 / N$ expansion one will have to tune the coefficients of all operators allowed by symmetries, including nonrenormalizable ones (Of course, we do not need to tune these infinite number of coefficients independently since there would be an entire submanifold of trajectories which passes close to the gaussian 
UV and ends in the interacting IR). Note that at large $N$ we are still close to the free fixed point at the cutoff scale, but we do not start from it in the UV. Duality is thus a weaker concept, since we do not know precisely the Lagrangian at the cutoff.

An example (not necessarily the specific theory we have discussed in the paper so far) of how small subleading $1 / N$ effects may change the global structure of the flow, and the need to fine tune at the UV, is shown in fig. 1 .

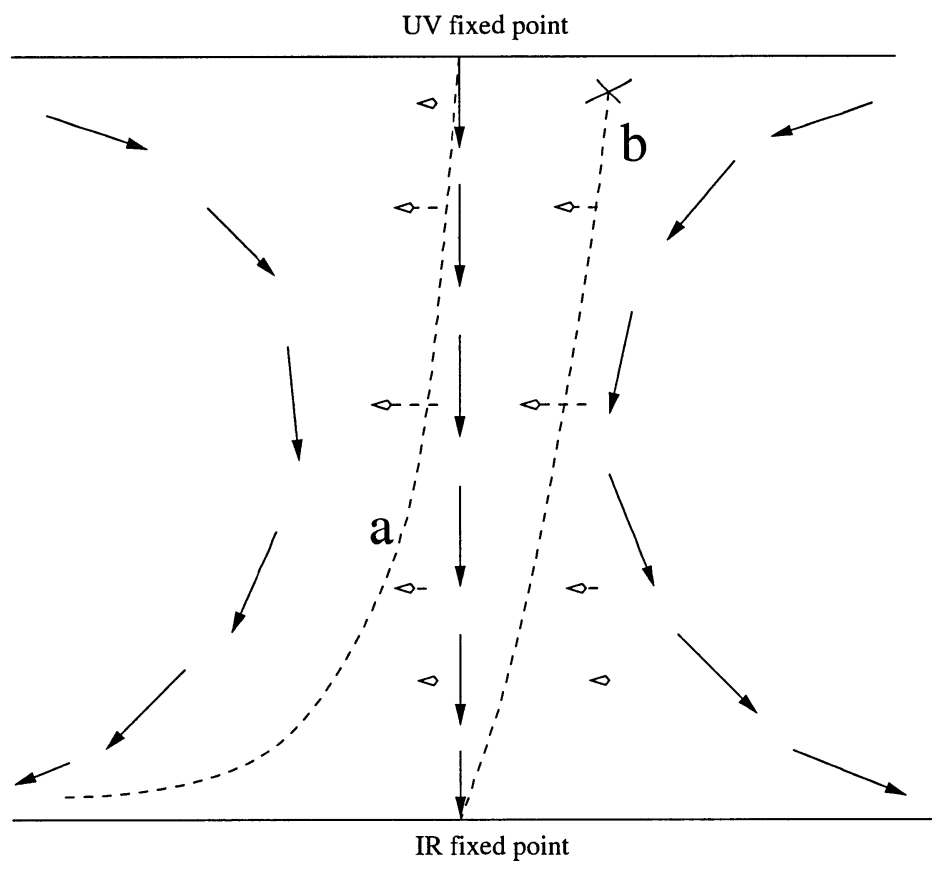

Figure 1: Global aspects of the flow. Black arrows are the leading N contribution. Dashed/White arrows are the subleading $\mathrm{N}$ correction. Line $\mathrm{a}$ is the modified flow from the UV fixed point. Line $\mathrm{b}$ is the fine tuned trajectory needed to hit the IR fixed point (we have neglected the fact that the IR fixed point moves a bit once $1 / N$ corrections are included.

\subsubsection{M-theory considerations}

In the $A d S / C F T$ correspondence the statement that for $N=\infty$ the $\mathrm{RG}$ flow is the same as in $\mathcal{N}=8$ is mimicked by the fact that the 
orbifold of the entire $\mathcal{N}=8$ solution [10] at all scales is still a solution of the classical equations of motion.

Consider now $1 / N$ corrections. These corrections are present at each value of $U$ (where $U$ is the additional coordinate in the $A d S$, which contains information about the RG flow). The zeroth order solution is no longer a solution and we need to correct it. When correcting it we may either keep the boundary conditions at $U=\infty$ fixed or the behavior at $U=0$ fixed. In the first case we keep the UV of the theory fixed but then the corrections at $U=0$ may be significant and the solution there may longer by approaching $A d S$. Instead we would like to keep the $A d S$ near $U=0$ but we can do so at the price of maybe changing the $U=\infty$ behavior.

One may ask whether from the supergravity description one can argue that the field theory becomes a gaussian theory in the UV. It would seem that the answer is no. The reason is that in the supergravity solution all that one sees near the boundary of the space-time are large curvatures [10]. Without independent means of computing at large curvature, all one can say is that this is consistent with the field theory becoming weakly coupled in the UV. One may perhaps also deduce the number of degrees of freedom from black hole entropy counting, or other dominant effects, but one can not argue that one knows exactly the Lagrangian of this weakly coupled theory at some given cutoff.

\subsection{An example of a nonsupersymmetric dual pair}

We need to exhibit two distinct theories which flow in the IR to the theory of anti-M2 branes near the B-singularity. For example, we may consider $\left(\mathbb{R}^{7} \times \mathbf{S}^{1}\right) / \mathbb{Z}_{2}$ orbifolds of M-theory of types $\mathrm{AB}$ and $\mathrm{BB}$ and probe them with anti-M2-branes. At weak coupling (i.e. when the radius of $\mathbf{S}^{1}$ is small) the M-theory orbifold of type BB becomes an $\widetilde{O 2}^{+}$ plane in IIA, while the orbifold of type $\mathrm{AB}$ becomes an $\mathrm{O}^{+}$plane. AntiM2 branes become anti-D2 branes in this limit. Naively, one expects the theories of anti-D2 branes probing the $\widetilde{\mathrm{O}}^{+}$and $\mathrm{O}^{+}$planes to be IR dual. As explained above, this is only literally true for $N=\infty$, and for finite $N$ one may need to add renormalizable and nonrenormalizable operators with fine-tuned coefficients in order to preserve duality. An 
analogous supersymmetric duality was suggested in [17]. The difference is that in the supersymmetric case the theories have a moduli space of vacua, and to see the duality one needs to go to a specific place in the moduli space. We have argued above that in the nonsupersymmetric case the moduli space is lifted at subleading order in the $1 / N$ expansion, so both theories have a unique vacuum and no tuning of the moduli is necessary.

The theories on anti-D2 branes are of course gauge theories. They are closely related to $\mathcal{N}=8$ theories on D2 branes probing the same backgrounds; in fact, the bosonic fields are identical. To obtain the spectrum of fermions recall that the field theory on $N$ (anti-)D2 branes near an orientifold 2-plane is obtained by orientifolding the spectrum of the $\mathcal{N}=8 U(2 N)$ theory. In the supersymmetric case the projection is identical for fermions and bosons, while in the nonsupersymmetric case the projection for the fermions has an extra minus sign compared to that for the bosons. It follows that the spectrum of the gauge theory of $\mathrm{N}$ anti-D2 branes near an $\widetilde{\mathrm{O}}^{+}{ }^{+}$(resp. $\mathrm{O}^{+}$) orientifold contains gauge bosons and seven real scalars in the adjoint of $S O(2 N+1)$ (resp. $S p(2 N)$ ) and eight Majorana fermions in the symmetric tensor representation of $S O(2 N+1)$ (resp. antisymmetric tensor representation of $S p(2 N))$. We do not know the precise Lagrangian, for reasons explained above. At leading order in $1 / N$ the Lagrangian can be obtained by taking the corresponding $\mathcal{N}=8$ Lagrangian describing D2 branes and replacing fermions in the adjoint by fermions in the appropriate tensor representation of the gauge group. This Lagrangian is superrenormalizable. We expect that all terms allowed by symmetries, including nonrenormalizable ones, would have to be included at next-to-leading order if one wants to flow to the CFT described by the "skew-whiffed" $A d S^{4} \times \mathbb{R P}^{7}$.

\section{Acknowledgments}

We would like to thank O. Aharony, S. Kachru, E. Silverstein, and M. Strassler for useful discussions. The work of MB is supported by NSF grant PHY-9513835. The work of AK is supported by DOE grant DE-FG02-90ER40542. 


\section{References}

[1] O. Aharony, Y. Oz, and Z. Yin, $M$-Theory on $A d S(P) \times S(11-P)$ and Superconformal Field Theories, Phys.Lett., B430 (1998), 87, hep-th/9803051.

[2] T. Banks and A. Zaks, On the Phase Structure of Vector-Like Gauge Theories with Massless fermions, Nucl. Phys., B196 (1982), 189.

[3] M. Berkooz and A. Kapustin, New IR Dualities in Supersymmetric Gauge Theories, hep-th/9810257, to be published in JHEP.

[4] M. Berkooz and A. Kapustin, work in progress.

[5] M. Berkooz and S.-J. Rey, Nonsupersymmetric Stable Vacua of M-theory, JHEP, 9901 (1999), 014, hep-th/9807200.

[6] M. Bershadsky, Z. Kakushadze and C. Vafa, String Expansion as Large N Expansion of Gauge Theory, Nucl. Phys., B523 (1998), 59, hep-th/9803076;

Z. Kakushadze, Gauge Theories from Orientifolds and Large $N$ Limit, Nucl. Phys., B529 (1998), 157, hep-th/9803214;

M. Bershadsky and A. Johansen, Large N Limit of Orbifold Field Theories, Nucl. Phys., B536 (1998), 141, hep-th/9803249.

[7] B. Biran, A. Casher, F. Englert, M. Rooman, and P. Spindel, The Fluctuating Seven Sphere in Eleven Dimensional Supergravity, Phys. Lett., 134B (1984), 179;

L. Castellani, R. D'Auria, P. Fre, K.Pilch, and P. Van Nieuwenhuizen, The Bosonic Mass Formula for Freund-Rubin Solution of $d=11$ Supergravity General Coset Manifolds, Class. Quant. Grav., 1 (1984), 229.

[8] M.J. Duff, B.E.W. Nilsson, and C.N. Pope, Phys. Rep., 130 (1986), 1;

M.J. Duff, B.E.W. Nilsson, and C.N. Pope, Spontaneous Supersymmetry Breaking by the Squashed Seven-Sphere, Phys. Rev., 50 (1983), 2043; Erratum, 51 (1983), 846. 
[9] A. Fayyazuddin and M. Spalinski, Large N Superconformal Gauge Theories and Supergravity Orientifolds, Nucl. Phys., B535 (1998), 219, hep-th/9805096;

O. Aharony, A. Fayyazuddin, and J. Maldacena, The Large $N$ Limit of N=2,1 Field Theories from Threebranes in F-Theory, JHEP, 9807 (1998), 013, hep-th/9806159;

A. Kehagias, New Type IIB Vacua and Their F-Theory Interpretation, Phys. Lett., B435 (1998), 337, hep-th/9805131.

[10] N. Itzhaki, J. Maldacena, J. Sonnenschein, and S. Yankielowicz, Supergravity and the Large $N$ Limit of Theories with Sixteen Supercharges, Phys. Rev., D58 (1998), 46, hep-th/9802042.

[11] S. Kachru and E. Silverstein, 4-D Conformal Theories and Strings on Orbifolds, Phys. Rev. Lett., 80 (1998), 4855, hep-th/9802183.

[12] I.R. Klebanov and A.A. Tseytlin, D-Branes and Dual Gauge Theories in Type 0 String, hep-th/9811035;

I.R. Klebanov and A.A. Tseytlin, Asymptotic Freedom and Infrared Behavior in the Type 0 String Approach to Gauge Theory, hep-th/9812089;

I.R. Klebanov and A.A. Tseytlin, A Nonsupersymmetric Large $N$ CFT from Type 0 String Theory, hep-th/9901101;

A.A. Tseytlin and K. Zarembo, Effective Potential in NonSupersymmetric $S U(N) \times S U(N)$ Gauge Theory and Interactions of Type 0 D3-Brane, hep-th/9902095.

[13] J. Maldacena, The Large N Limit of Superconformal Field Theories and Supergravity, Adv. Theor. Math. Phys., 2 (1998), 231, hep-th/9711200.

[14] J. Maldacena, J. Michelson, and A. Strominger, Anti-de-Sitter Fragmentation, hep-th/9812073.

[15] N. Nekrasov and S.L. Shatashvili, On Nonsupersymmetric CFT in Four Dimensions, hep-th/9902110.

[16] N. Seiberg, Notes on Theories with Sixteen Supercharges, Nucl. Phys. Proc. Suppl., 67 (1998), 158-171, hep-th/9705117.

[17] S. Sethi, A Relation between $N=8$ Gauge Theories in Three Dimensions, JHEP, 9811 (1998), 003, hep-th/9809162. 
[18] E. Witten, Baryons and Branes in AdS, JHEP, 9807 (1998), 006, hep-th/9804001.

[19] E. Witten, Anti-de-Sitter Space and Holography, Adv. Theor. Math. Phys., 2 (1998), 253, hep-th/9802150;

S.S. Gubser, I.R. Klebanov, and A.M. Polyakov, Gauge Theory Correlators from Noncritical String Theory, Phys. Lett., B 428 (1998), 105, hep-th/9802109. 